\title{
Efficient Polymer Scattering Layers Fabrication and their Applications in Electrical Properties Enhancement of Perovskite/Silicon Tandem Solar Cells
}

\author{
ASAD Ali ${ }^{1, a, *}$, SADDAM Ali ${ }^{1, b}$, HASSAN Ali ${ }^{2, c}$, KAMRAN Alam $^{1, d}$, \\ WAQAR Ali $^{1, \mathrm{e}}$, NOAMAN Khan ${ }^{1, \mathrm{f}}$, SALMAN Manzoor ${ }^{2, \mathrm{~g}}$, \\ ZACHARY Holman ${ }^{3, \mathrm{~h}}$ and MUHAMMAD Arif ${ }^{1, \mathrm{i}}$
}

\author{
${ }^{1}$ United States-Pakistan Center for Advanced studies in Energy, University of Engineering and \\ Technology, Peshawar, 25000, Pakistan \\ 2Department of Physics, Government Post Graduate College Nowshera, 24600, Pakistan \\ ${ }^{3}$ Arizona State University, Tempe, Arizona, 85281, USA \\ a*asadali3201@gmail.com, bsak2581992@gmail.com, chali71736@gmail.com, \\ dkamranalam203@gmail.com, ${ }^{\mathrm{e}}$ wiqar1@gmail.com, ${ }^{\mathrm{f}}$ nomankhanmech@gmail.com, \\ gsalman.manzoor86@gmail.com, hzachary.holman@asu.edu, 'Muhammad.arif@hotmail.com
}

Keywords: Light Trapping, Transmittance, Reflectance, Tandems, Anti-reflection Coatings.

\begin{abstract}
Tandem solar cells with silicon as one of its constituents have flat surfaces (surfaces without texturing). That is why flat surfaces Solar cells have got quite importance. But the issue with the flat surfaces is the high reflection loss (flat) and poor light trapping (no-texturing) in the cells. So, some scattering film, other than direct texturing, that is polydimethylsiloxane (PDMS) polymer with the texture is used. The optimized PDMS film here is the random pyramidal film because random pyramidal PDMS films have a drop of $56.6 \%$ (from 33.54 to $14.48 \%$ ) in reflectance used on polished Silicon while iso-textured and inverted pyramids have 51.55\% (33.54 to $16.25 \%$ ) and $48.47 \%$ (from 33.54 to $17.28 \%$ ) respectively. This PDMS film with random textures when applied to 2-terminal monolithic perovskite/Silicon tandem, its external quantum efficiency shows an increase of $1.16 \mathrm{~mA} / \mathrm{cm}^{2}$ in the short-circuit current and reflection loss reduces by $4.1 \mathrm{~mA} / \mathrm{cm}^{2}$.
\end{abstract}

\section{Introduction}

The primary function of a solar cell device is to convert all the photons hitting its surface to electricity. However, the front (sunward facing) surface of a solar cell is a source of reflectance loss due to the mismatch of refractive-index between air and the absorber material, or the absorber material and the encapsulant material in the case of modules-Fresnel reflection losses. There are methods by which reflection from Solar cells is reduced by having light to bounce on the front surface more than once thus increasing the chances to transmit more light into the Solar cells. Furthermore, such techniques make light to take a longer path inside the Solar cells and thus allowing weakly absorbed light with more distance to get absorbed. Various methods have been used for this purpose, thus even when the wafer is thin the weakly absorbed light can be totally internally reflected many times between front and back surface to have better chance of absorbing it in Solar cells rather than escaping from Solar cells. One method to reduce front surface reflection is the use of anti-reflective coating (ARC) mainly of Magnesium Flouride and porous Silicon Oxides $[1,2]$. Another method to reduce the front surface reflection is to use structures which are antireflecting [3], that lower the refractive index at interface between air and glass surfaces by the use of either a porous or a textured glass on a sub-wavelength scale. One other method is the use of anti-reflective textures that are geometric that allow multiple rebounding of the incident light thus increasing the transmittance through the glass to the active layers. Its advantage is that the antireflective effect has spectral independence. In mono-crystalline silicon the front of the wafer is textured [4] by etching in $\mathrm{KOH}$ alkaline solution with resulting texture of square based pyramids. 
The same kind of etching in acidic solution for multi-crystalline silicon results in the front surface with spherical-cap shaped textures (iso-textures) [5]. On crystalline Silicon another similar texturing is done by interference lithography technique with subsequent dry and $\mathrm{KOH}$ wet etching that results in textures with inverted pyramids [6]. Similarly, subwavelength structures (SWS) are fabricated on the surface of solar cells to mitigate the optical losses using different techniques. These SWSs vary in structure such as nano-dot mask, moth-eye, nano-rod and nano-structures etc, and shape such as triangular, tapered, conical etc [7]. While textured substrates like textured $\mathrm{ZnO}$ are used to grow thin film solar cells on them $[8,9]$. Thus different methods have been employed for mitigating the optical losses from the surface of solar cells and to trap more and more light, thus to increase efficiency. But the techniques used for fabrication of such structures or layers are costly and complicated and thus are limited to only small area applications [10]. Similarly, the processes for fabricating multi-layer anti-reflective layers are high temperature, high cost and instable which limit their applications [11]. For many other solar cells, however, it is inconvenient or impossible to texture the absorber layer. For example, the best perovskite solar cells are presently deposited from solution, and attempts to accommodate non-planar substrates that would impart their texture to the perovskite layer have thus far decreased conformity and degraded cell performance [12]. III-V cells are grown epitaxially on polished, lattice-matched substrates, and etching the absorber to define scattering features both would be impractical and would inhibit subsequent growth of epitaxial passivation and contact layers [13]. Even polycrystalline thin-film solar cells, like a CIGS and $\mathrm{CdTe}$, are commonly grown on near-planar substrates and do not themselves develop much texture during growth; growth of polycrystalline films on highly textured substrates can create voids in the material, resulting in poor device performance or even shunting [14]. Reflective flat surfaces are also not unique to single-junction cells. All modules with planar front glass incur a minimum 4\% reflectance loss at the air/glass interface for normally incident light, regardless of the reflectance of the cells encapsulated therein. In addition, tandem cells (and modules) are rapidly improving as concepts pioneered in III-V multi-junction devices and thin-film silicon tandems are adapted to, e.g., top-cell/Si tandems and perovskite/perovskite tandems [15], yet most have flat front surfaces and thus reflection is a primary efficiency limiter $[16,17]$. This loss can be particularly detrimental in two-terminal tandems, as reflection is often not constant across the full spectrum and thus influences current matching [18]. One way to deal with this optical loss in flat devices is to have a scattering surface on top of the front surface of the device, and in case of modules, texture the front surface of glass as proposed in $[8,19]$. In this contribution we show that a polymer based scattering layer, polydimethylsiloxane (PDMS), carrying random pyramidal texture could be used as such a scattering surface. PDMS with textures from three masters are compared and the best, random, is then applied to the front of a tandem solar cell which is a 2-terminal monolithic perovskite/silicon device and its electrical properties are measured which resulted an increase in the $\mathrm{J}_{\mathrm{SC}}$.

\section{Optimized PDMS Scattering Layers}

A nano-imprinting Lithography (NIL) technique, soft lithography, is used for the transfer of the nano-metric structured textures from the Silicon masters on a polymer polydimethylsiloxane (PDMS). The replication of the original surface textures was made from silicon wafers that contained the inverse of the texture being replicated on the PDMS. PDMS scattering layers were made with Sylgard 184, from Dow Corning. The process involved mixing of base and curing agent in 10:1 ratio and then the mixture was degassed in the vacuum before dispensing it on silicon wafers that already carried textures. This degassed mixture was cured at a temperature of $46{ }^{\circ} \mathrm{C}$ for 12 hours. In the end, PDMS was carefully peeled off the silicon wafer and it then had the negative of the textures on the master wafers [20]. Three types of masters were used with three different types of textures: random pyramids, inverted pyramid textures and iso-textures. The PDMS scattering layers were fabricated using these three different masters and were compared on the basis of their optical performance. These layers were applied to plane (un-textured polished) Silicon and their reflectance was measured with Perkin-Elmer 950-Lambda Spectrophotometer. Similarly, the transmittance and reflectance for the individual PDMS layers with these textures were measured. 
The comparison showed the optimized, efficient and best textured PDMS was one with random pyramids. Fig. 1 shows the comparison of the three samples. The total reflectance of the three masters is shown in Fig. 1 (a). It shows that the reflectance for random is higher as compared to the other two masters, for the iso-texture master it is due to the anti-reflective coatings on the surface of the wafer. As it is seen that throughout the spectrum from $250 \mathrm{~nm}$ upto $1000 \mathrm{~nm}$ the reflectance is lower than the other two masters. In the $500-700 \mathrm{~nm}$ region the reflectance for inverted textures is lower than the rest of masters. The textures from these masters when transferred to PDMS and then applied to polished silicon wafer showed PDMS with random textures as the best among the three; it also reduced the reflectance of the polished silicon by $56.6 \%$ from 33.54 to $14.48 \%$ when random textured PDMS was applied to polished silicon, $48.47 \%$ for inverted textures from 33.54 to $17.28 \%$, $51.55 \%$ for iso-texture from 33.54 to $16.25 \%$. The comparison is shown in figure 1 (b).
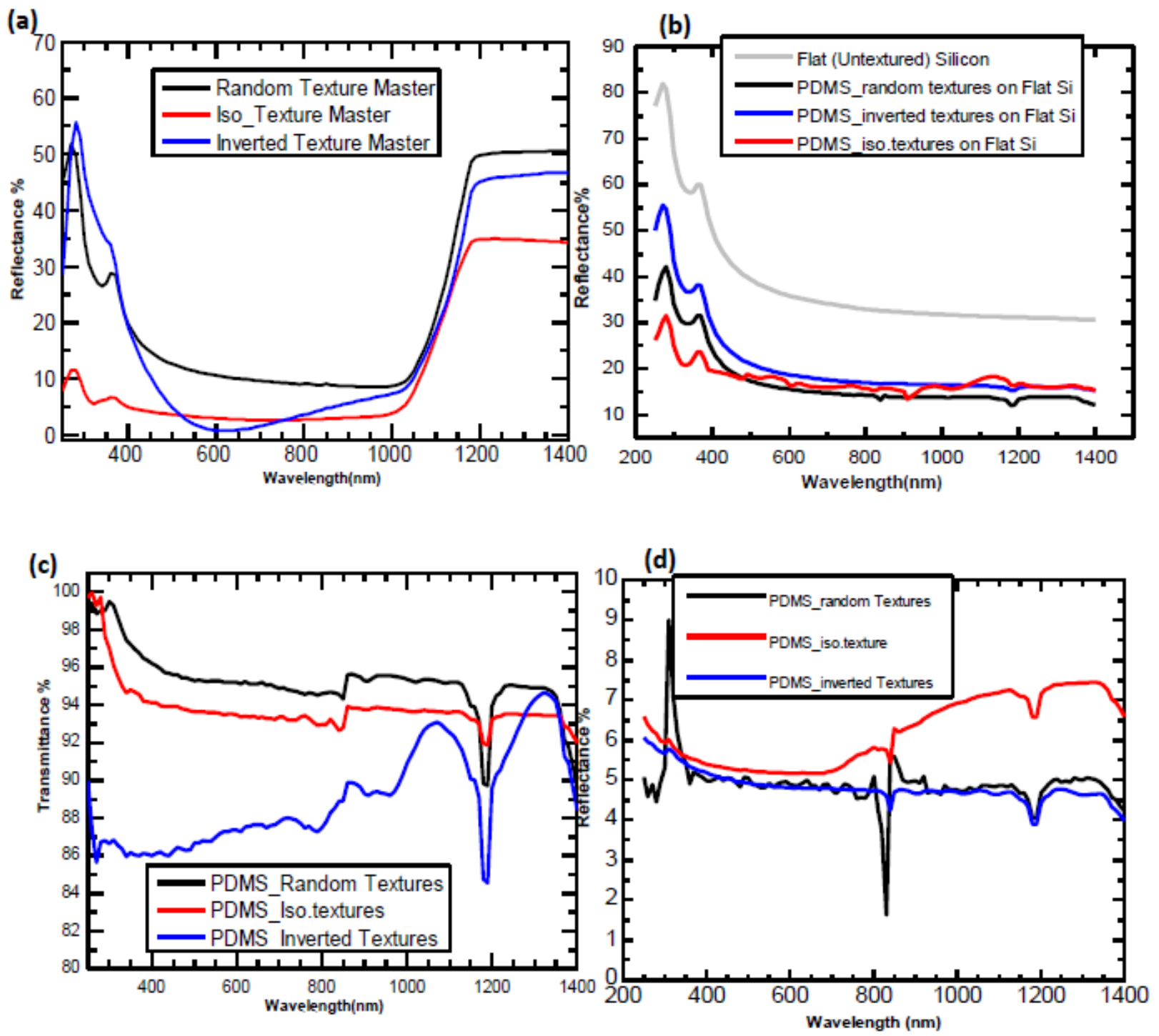

Fig. 1. (a) Total reflectance of the three masters (b) Reflectance of PDMS layers with three textures on Polished Silicon vs Polished Silicon (c) Transmittance of the three PDMS layers (d) Reflectance of the three PDMS layers

Another striking quality of the PDMS with random pyramids is its transmittance and reflectance reduction. Fig. 1 (c) shows the transmittance curves for the PDMS with the three textures, which has random curve on the top with higher transmittance as compared to the other two. While Fig. 1 (d) shows the comparison of reflectance for the three samples. Again the reflectance for the random is lower compared to the iso-texture and comparable with the inverted. These results depict PDMS with random textures as the best among the three due to efficient transfer of textures from the respective master as compared to the other two. Further, the morphology of the PDMS with this 
texture is studied such as atomic force microscopy (AFM) and scanning electron microscopy (SEM).

\section{Textured PDMS Morphology}

Fig. 2 (a) shows the AFM image for the bare silicon without any PDMS textured layer on it [21]. This silicon wafer has random pyramidal textures from the $\mathrm{KOH}$ etching. The heights of pyramids vary from $2-5 \mu \mathrm{m}$ (random). This image is taken on an area of $20 \mu \mathrm{m} X 20 \mu \mathrm{m}$ on the silicon wafer. Fig. 2 (b) shows the two dimensional AFM image of the PDMS scattering layer that is fabricated with the textures on it. This image is taken on an area of $140 \mu \mathrm{m} \mathrm{X} 75 \mu \mathrm{m}$. The heights of the pyramids in the replica are small comparative to the masters so the two dimensional AFM image could not give the details. The image is processed with nanoscope software, AFM Bruker software, and the three dimensional image is taken, that shows the heights as well, Fig. 2 (c).

(a)

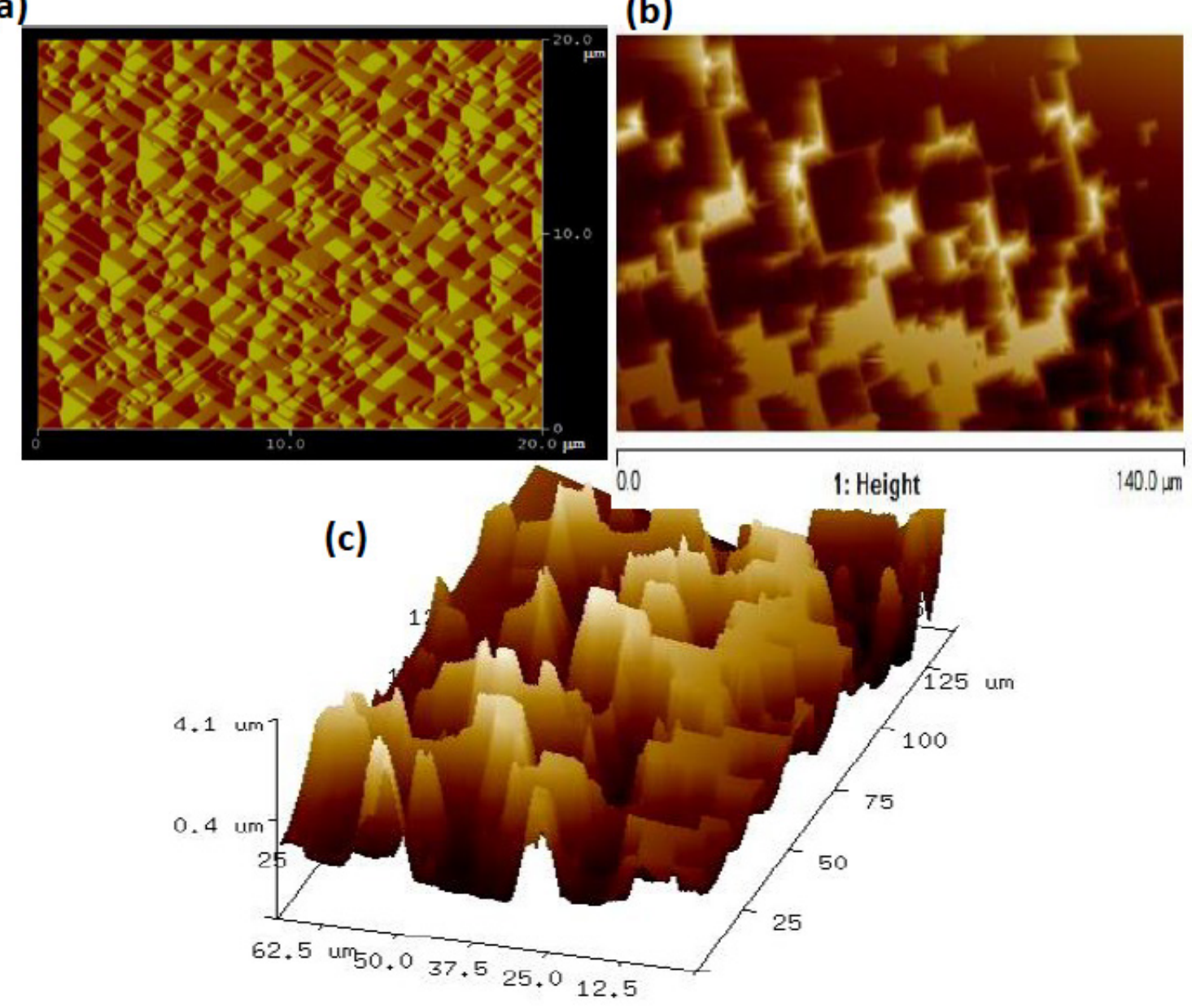

Fig. 2. (a) AFM image on $20 \mathrm{X} 10 \mu \mathrm{m}^{2}$ of silicon wafer with random textures [21] (b) 2D AFM image on $140 X 75 \mu \mathrm{m}^{2}$ of PDMS with same random textures (c) 3D AFM image of the PDMS with $4.1 \mu \mathrm{m}$ height

Fig. 3 (a) shows the SEM image of silicon master wafer with random textures on an area of $20 \mu \mathrm{m}^{2}$. Similar results had been published in earlier publication [22]. It clearly shows the pyramids of various sizes with respect to their heights and base angles. Fig. 3 (b) shows the SEM image of PDMS scattering layer with the replica of textures from the master silicon on an area of $10 \mu \mathrm{m}$, small area because the replica is not as efficient as direct texturing the wafers. There are different regions in the SEM image of PDMS. The ridges show the upright pyramids while the black small holes show downright pyramids in to the surface of PDMS - random pyramids. 

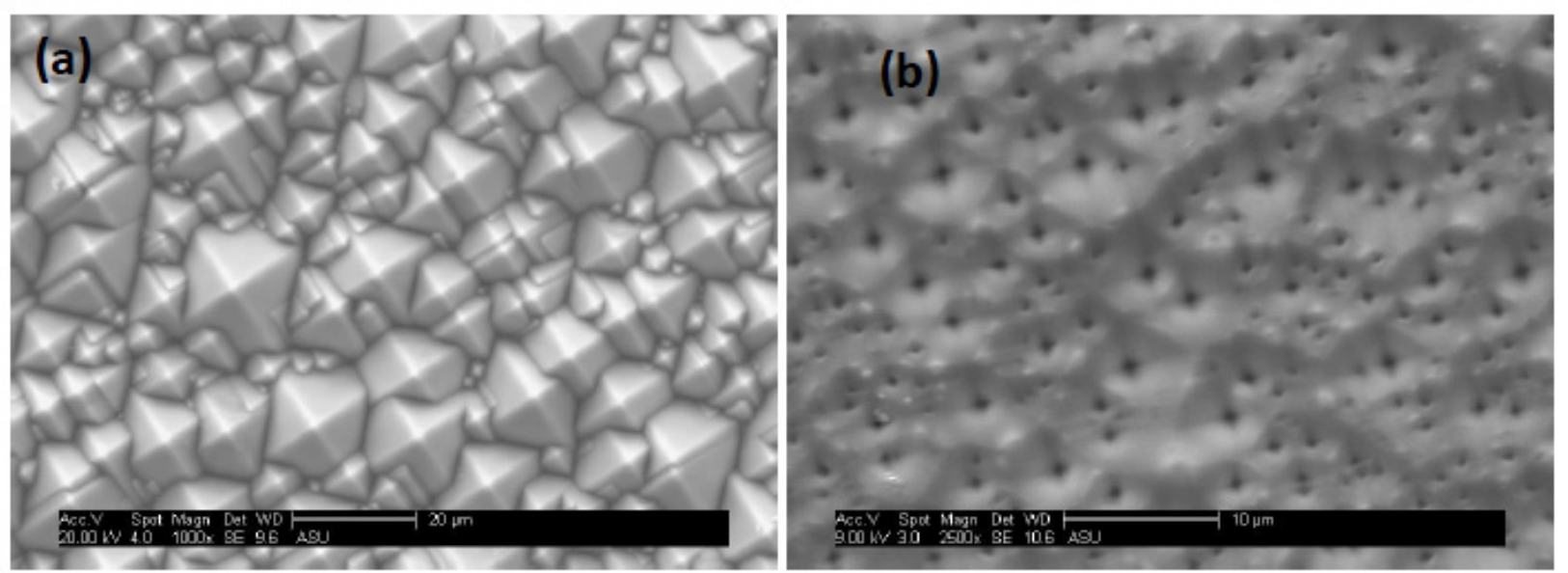

Fig. 3. (a) SEM image of Silicon Wafer (b) SEM image of PDMS texture [22]

\section{PDMS on Perovskite/Silicon Tandem.}

The best achieved PDMS scattering layer with random pyramidal texture was then applied to perovskite/silicon tandem solar cell. The PDMS was applied first to flat silicon devices and perovskite, as has been published in [22] with increase of $\mathrm{J}_{\mathrm{SC}}$ for the silicon and perovskite devices $1.7 \mathrm{~mA} / \mathrm{cm}^{2}$ and $1.9 \mathrm{~mA} / \mathrm{cm}^{2}$, respectively. For the fabrication of 2-terminal perovskite/silicon tandem [16] the top cell was perovskite fabricated on top of silicon as bottom cell, Fig. 4 (a). For prevention of deterioration in the passivated surface layers of the bottom silicon cell a different annealing conditions were applied. The annealing temperature and time used were $190{ }^{\circ} \mathrm{C}$ and $1 \mathrm{hr}$ for the NiOx layer instead of $300{ }^{\circ} \mathrm{C}$ and $1 \mathrm{hr}$, as was for the single junction perovskite.

(a)

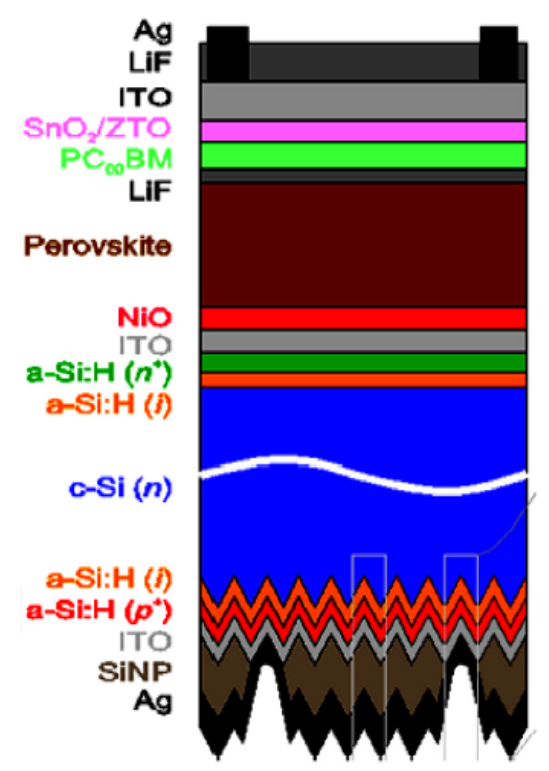

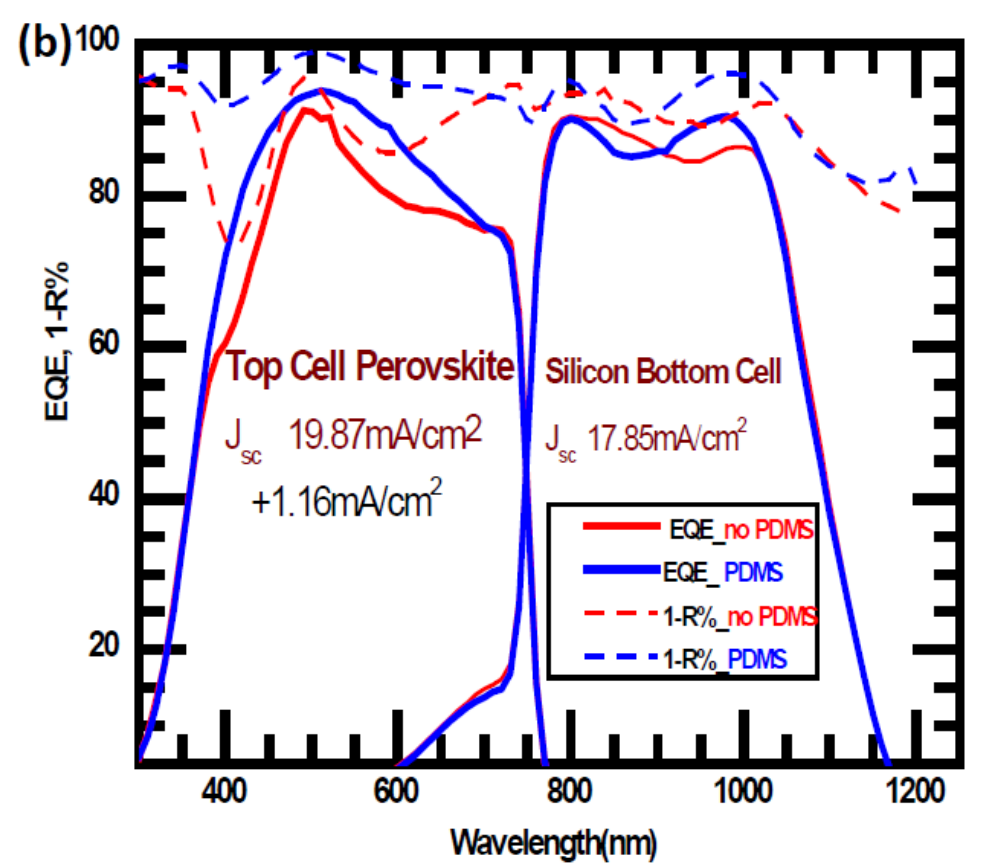

Fig. 4. (a) 2-terminal monolithic perovskite/silicon tandem device (b) EQE and 1-R\% for the tandem with and without PDMS scattering layer

The EQE measured for the tandem device with and without the PDMS scattering layer is shown in Fig. 4 (b). The blue curve shows EQE and 1-R\% for the device with PDMS and the red without PDMS. In the left portion of the graph it is observed that blue curve overlaps the red from $250 \mathrm{~nm}$ to approximately $700 \mathrm{~nm}$ that results in an increase in the $\mathrm{J}_{\mathrm{SC}}$ of $1.16 \mathrm{~mA} / \mathrm{cm}^{2}$ and the reflection loss reduces by $4.1 \mathrm{~mA} / \mathrm{cm}^{2}$ for Perovskite top cell. Looking in to the right portion of the EQE curve there is cross-overlapping of the blue and red curves for the silicon bottom cell of the tandem configuration. 


\section{Conclusion}

Solar cells which cannot be textured, directly, suffer from large optical losses due to high front surface reflectance and poor light trapping of weakly absorbed long wavelengths. In this contribution we have demonstrated that polymer scattering layers (PDMS) when applied to the front surface of flat solar devices successfully reduce front reflectance loss.

\section{Acknowledgement}

This work is based on the research carried out at Materials for Energy lab, Arizona State University, USA. This is supervised by Zachary Holman, Assistant Professor, and Salman Manzoor, Ph. D. scholar.

\section{References}

[1] S. Chattopadhyay, Y.F. Huang, et al, Anti-reflecting and photonic nanostructures, Mater. Sci. Engineer., 69 (2010) 1-35.

[2] C. Ballif, J. Dicker, et al, Solar glass with industrial porous $\mathrm{SiO}_{2}$ antireflection coating: measurements of photovoltaic module properties improvement and modeling of yearly energy yield gain, Sol. Ener. Mater. Sol. Cel., 82 (2004) 331-344.

[3] P.K. Chang, P.-T. Hsieh, et al, Improvement of the short-circuit current density and efficiency in micromorph tandem solar cells by an anti-reflection layer, Th. Sol. Fil., 520 (2011) 550-553.

[4] J.D. Hylton, et al, Alkaline etching for reflectance reduction in monocrystalline silicon solar cells, J. Electrochem. Soc., 151 (6) (2004) G408-G427.

[5] K.R. McIntosh, T.G. Allen, et al, Light trapping in iso-textured silicon wafers, IEEE J. Photovolt. 7 (2017) 110-117.

[6] S.Sivasubramaniam, M.M. Alkaisi, Inverted nanopyramid texturing for silicon solar cells using interference lithography, 2014, Published by Elsevier B.V. Microelectronic Engineering.

[7] J.Y. Chen, K.W. Sun, Enhancement of the light conversion efficiency of silicon solar cells by using nanoimprint anti-reflection layer, Sol. Ener. Mater. Sol. Cel., 94 (2010) 629-633.

[8] J. Escarre, K. Söderström, et al, Geometric light trapping for high efficiency thin film silicon solar cells, Sol. Ener. Mater. Sol. Cel., 98 (2012) 185-190.

[9] M. Jošt, S. Albrecht, et al, Back-and Front-side Texturing for Light-management in Perovskite/Silicon heterojunction Tandem Solar Cells, Energ. Proced., 102 (2016) 43-48.

[10] C.J. Ting, M.C. Huang et al, Low cost fabrication of the large-area anti-reflection films from polymer by nanoimprint/hot-embossing technology, Nanotechnol., 19(2008) 205301 (5pp).

[11] J. K. Tsai and Y. S. Tu, Fabrication of Polymeric Antireflection Film Manufactured by Anodic Aluminum Oxide Template on Dye-Sensitized Solar Cells, Mater., 10 (2017) 296-302.

[12] W. Jaegermann, A. Klein, T. Mayer, Interface engineering of inorganic thin-film solar cellsmaterials-science challenges for advanced physical concepts, Adv. Mater. 21 (2009) 4196-4206.

[13] A.R. Pascoe, S. Meyer, et al, Enhancing the optoelectronic performance of perovskite solar cells via a textured CH3NH3PbI3 morphology, Adv. Funct. Mater. 26 (2015) 1278-1285.

[14] J. Fritsche, S. Gunst, et al, Surface analysis of CdTe thin film solar cells, Th. Sol. Fil., 387 (2001) 161-164.

[15] M. Umeno, et al, Hetero-epitaxial technologies on Si for high-efficiency solar cells, Sol. Energy Mater. Sol. Cel., 50 (1998) 203-212. 
[16] K.A. Bush , A.F. Palmstrom , Z.J. Yu et al, 23.6\% efficient monolithic perovskite/silicon tandem solar cells with improved stability, Nat. Energ., 2 (2017) 17009.

[17] S. Albrecht, M. Saliba, J.P.C. Baena, F. Lang, L. Kegelmann, M. Mews, L. Steier, A. Abate, J. Rappich, L. Korte, Monolithic perovskite/silicon-heterojunction tandemsolar cells processed at low temperature, Energ. Environ. Sci., 9 (2016) 81-88.

[18] X. Wang, A. Barnett, The effect of spectrum variation on the energy production of triplejunction solar cells, IEEE J. Photovolt., 2 (2012) 417-423.

[19] M. Jošt, S. Albrecht, B. Lipovšek, J. Krč, L. Korte, B.Rech, and M. Topič, Back-and Front-side Texturing for Light-management in Perovskite/Silicon heterojunction Tandem Solar Cells, Energ. Proced., 102 (2016) 43-48.

[20] J. Escarre, K. Soederstroem et al, Highfidelity transfer of nanometric random textures by UV embossing for thin film solar cells applications. Sol. Ener. Mater. Sol. Cel., 95 (2011) 881-886.

[21] MS Thesis, Light Trapping in Monocrystalline Silicon Solar Cells Using Random Upright Pyramids by S. Manzoor at Arizona State University, USA, 2014.

[22] S. Manzoor, J. Yu Zhengshan, A. Ali et al, Improved light management in planar silicon and perovskite solar cells using PDMS scattering layer, Sol. Ener. Mater. Sol. Cel., 173 (2017) 59-65. 